\title{
Analysis Of Human-Induced Vibrations In A Lightweight Framework
}

\author{
A. de-Juan, P. Garcia, A. Fernández del Rincón, A. Diez-Ibarbia, M. \\ Iglesias, F. Viadero \\ Department of Structural and Mechanical Engineering, University of Cantabria. Avda. \\ de los Castros s/n 39005 Santander, Spain.
}

\begin{abstract}
This article analyzes the vibratory behavior of a Material-Composed Sandwich (MCS) framework for residential buildings. It has been observed qualitatively that the use of this kind of framework leads to poor comfort levels. The goal of this study is to find out the sources of this lack of comfort, in order to suggest guidelines that can enhance the performance of the MCS framework, without jeopardizing its advantages with respect to the traditional frameworks.

To achieve this objective, an Experimental Modal Analysis (EMA) of a sample MCS framework has been carried out in order to determine the dynamic parameters. Then, a numerical Finite Element (FE) model of said sample MCS framework has been developed and adjusted with the results obtained in the experimental test. Based on this, a real-dimension MCS framework FE model has been built and the resultant behavior compared with that of a commonly used framework made of reinforced concrete. This comparison is finally used to assess the uncomfortable dynamic response of the MCS framework and to draw conclusions on the design guidelines in order to enhance the MCS framework vibratory behavior.
\end{abstract}

Keywords: Composite framework, Experimental modal testing, Finite element model, Vibration behavior, Building vibration comfort

Email address: \{ana.dejuan, pablo.garcia, alfonso.fernandez, alberto.diez, miguel.iglesias, fernando.viadero\}@unican.es (A. de-Juan, P. Garcia, A. Fernández del Rincón, A. Diez-Ibarbia, M. Iglesias, F. Viadero) 


\section{Nomenclature}

$E$ Young's modulus

$G_{x y}$ Shear modulus

$I$ Moment of bending inertia

$\Delta_{a d m}$ Maximum admissible static deflection

$\Delta_{d l}$ Static deflection due to dead load

$\Delta_{f r m}$ Total static deflection

$\Delta_{l l}$ Static deflection due to live load

$\nu_{x y}$ Poisson's ratio

$\omega_{n}$ Natural frequency at the $n$ mode

$\xi$ Modal damping ratio

EMA Experimental Modal Analysis

EPS Expanded polystyrene

FE Finite element

FRF Frequency response function

MAC Modal Assurance Criterion

MCS Material-Composed Sandwich

MMIF Multivariate Mode Indicator Function

RC Reinforced Concrete

\section{Introduction}

The healthy building concept is not only related to the health needs of the users and energetic efficiency, but also to comfort features. In a recent study (Bluyssen, 2010), some guidelines are given to develop indicators for healthy and comfortable buildings. Perception of vibration in buildings is an issue linked to discomfort and annoyance of the users (ISO 2631/2, 2003). In a paper by Whittle, recommendations are given on how to measure vibration annoyance in residential environments using previously suggested scales (Whittle et al., 2015). The vibration may be perceptible or not, depending on the kind of building, the activities and the position (standing, sitting, recumbent) of the people inside the building, the intensity and duration of the vibration, sound insulation (Ljunggren et al., 2014), etc. A building can be considered comfortable, from the point of view of vibrations, when there is no perception of them (Griffin, 1996). The Eurocode (2002) classifies the comfort of users related to deflections and vibrations as one of the serviceability limit states. 
The use of lightweight frameworks is increasingly important in the building industry because it reduces assembly and transport costs, amongst others. There is a tendency to significantly diminish the mass and thickness of the frameworks and to increase their spans. The structural damping also decreases due to the lack of vibration-absorbing materials. Moreover, these kinds of frameworks usually employ orthotropic materials, whose dynamic behavior varies significantly from isotropic ones (Altintas and Goktepe, 2007). All these factors can produce an inappropriate vibration response to daily activity loads, i. e., human walking forces, machinery, etc. This is the reason why the characteristics of new frameworks tend to shorten the natural frequencies to values close to the human activity range of frequencies. For this reason, human activity usually produces a loss of comfort to the users of these kinds of frameworks, which causes a feeling of lack of safety and affects their normal life. It might even affect the structural integrity of the building. Thus, it is not enough to perform a static analysis, but it is necessary to estimate the vibration response during the design process of new buildings (Mello et al., 2008) or during refurbishment (Augusztinovicz, 2012). Nevertheless, there is not a unique criterion for the vibration design of lightweight frameworks (Ljunggren, 2006). The reason is that it is a multifaceted problem which depends on framework properties (i. e. mass, stiffness and damping) and other factors related to human activity (use of the building, position and activity of the people, etc.)

In this article, a study is carried out of the vibration response of a new Material-Composed Sandwich (MCS) framework, whose mass is twenty times less than concrete and has been used in the construction of a two-story prototype building. It has been subjectively noticed that this MCS framework has a poor vibrational behavior in terms of comfort in the prototype building. The goal of this study is to find out the sources of this lack of comfort, in order to suggest guidelines that can enhance the performance of the MCS framework, without jeopardizing its advantages with respect to the traditional frameworks.

\section{Framework Description and Solution Methodology}

The framework is composed of a core of Expanded Polystyrene (EPS) and by skins of composite (glass fiber and epoxy), as is shown in Figure 1. Its dimensions are $5 \times 3 \times 0.2$ meters, and the thickness of the skins is 2.5 millimeters. As the dimensions are so large, a sample of the MCS 
framework is also used; whose dimensions are $1 \times 2 \times 0.2$ meters. This sample is a prototype, which was manufactured especially for this work. The mechanical characteristics of the materials, given by the manufacturer, are shown in Table 1. As can be seen, the material of the skins is orthotropic, and the material of the core is isotropic.

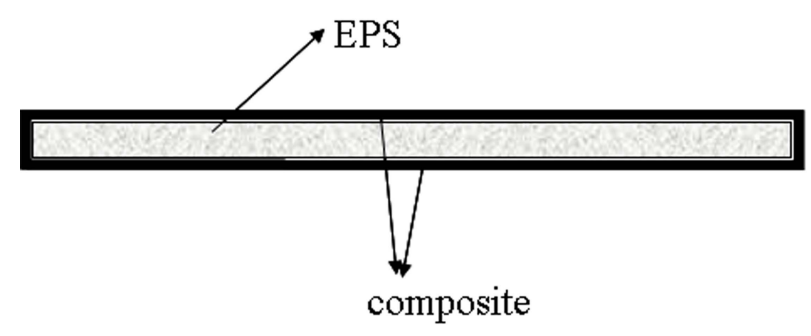

Figure 1: Description of the material-composed sandwich framework

The procedure to obtain a satisfactory solution to the vibration problem is detailed next. First of all, an Experimental Modal Analysis (EMA) of the sample MCS framework is carried out, where its dynamic parameters, namely, natural frequencies and modal damping ratio, are obtained. Then, a numerical Finite Element (FE) model of the sample MCS framework is developed and adjusted with the results obtained in the experimental test. Finally, a real-dimension MCS framework FE model, that will be used in the prototype building, is built. These results are compared with the ones obtained from a commonly used solution: reinforced concrete. This comparison will help in understanding the uncomfortable dynamic response of the MCS framework and lead to formulate conclusions that will enhance the original design.

Combining experimental modal and numerical $\mathrm{FE}$ analysis is a procedure widely used in structural engineering. Moreira and Rodrigues (2010) present a layerwise finite element, which considers the through-thickness deformation, for the analysis of isotropic and orthotropic laminates and they validate this formulation with experimental data. Matter et al. (2009) present an 
Table 1: Mechanical characteristics of the materials

\begin{tabular}{|c|c|c|}
\hline Property & Skins & Core \\
\hline$E_{x}$ & $15 \mathrm{GPa}$ & $15.5 \mathrm{MPa}$ \\
\hline$E_{y}$ & $15 \mathrm{GPa}$ & $15.5 \mathrm{MPa}$ \\
\hline$E_{z}$ & $2.5 \mathrm{GPa}$ & $15.5 \mathrm{MPa}$ \\
\hline$v_{x y}$ & 0.39 & 0.29 \\
\hline$G_{x y}$ & $2.5 \mathrm{GPa}$ & $6 \mathrm{MPa}$ \\
\hline Density & $1750 \mathrm{~kg} / \mathrm{m}^{3}$ & $70 \mathrm{~kg} / \mathrm{m}^{3}$ \\
\hline
\end{tabular}

interesting numerical-experimental method, which is based on modal information, for calculating elastic and damping parameters of composite multilayered plates and shells; results are obtained by minimizing discrepancies between numerical and experimental results. Pavić and Reynolds (2003) follow this technique to study the differences in the dynamic parameters between the cracked and uncracked states of a high-strength concrete framework. $\mathrm{Wu}$ (2004) builds a FE model of a scaled crane rig improved by correlation. Dooms et al. (2006) present a FE model of a silo correlated with experimental modal analysis in order to improve the design of the silo with respect to ovalling owing to wind. This procedure is also applied for the dynamic behavior of sandwich panels with a non-structural application. For instance, Petrone et al. (2014) show a similar procedure for modal characterization of sandwich panels used in interiors of road vehicles. Mucchi (2013) shows another study where beach tennis rackets, which are also composed innerly by sandwich panel, are dynamically characterized.

\section{Experimental Testing}

The main aim of experimental testing is to establish the dynamic (modal) properties of the framework, namely, natural frequencies, mode deformation patterns and modal damping ratios. These parameters will be used in the next section to check and correlate the numerical FE model, which will simulate the behavior of the real-dimensioned MCS framework.

Conditions for experimental testing are explained next. The sample was emplaced vertically by means of three bungee cords in order to achieve free body condition, as can be seen in Figure 2 .

The natural frequencies of the bungee cords are much lower than the lowest natural frequency of the MCS framework. Then, a grid of fifteen ex- 


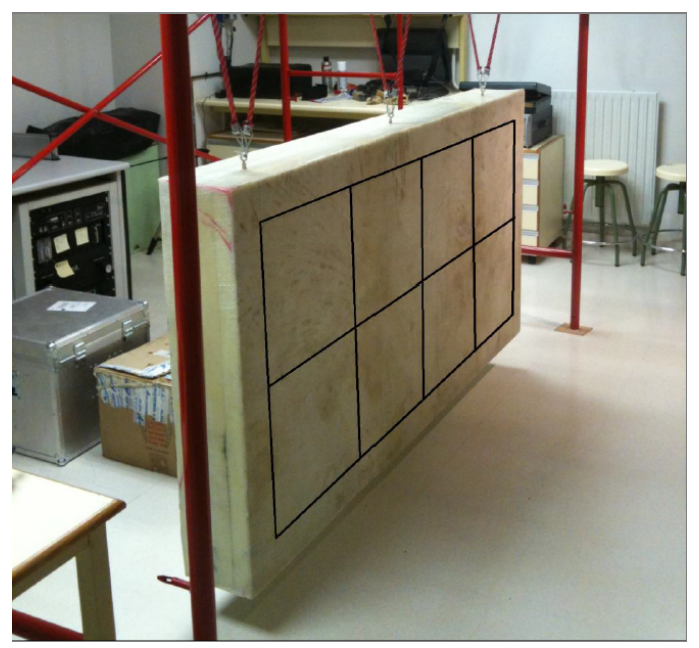

Figure 2: Boundary conditions and grid measurement of the sample MCS framework

citation points was defined. An impact hammer (PCB model 086B03) was used to excite the MCS framework in the normal direction to its surface on the grid points. A general purpose accelerometer (Brüel \& Kjær, type 4399) was fixed by adhesion in order to measure the acceleration. In this way, for a determined location of the accelerometer at one corner, the hammer was moved impacting in the rest of the grid points obtaining the corresponding Frequency Response Functions (FRF) by averaging ten impacts for each excitation point with a frequency range up to $640 \mathrm{~Hz}$. Both impact hammer and accelerometer were connected to LMS SCADAS III acquisition system and controlled by LMS. TestLab software.

Figure 3 shows the Multivariate Mode Indicator Function (MMIF) (Williams et al., 1985) for the first four modes. After the data acquisition was performed, modal parameters were extracted using the $i d_{-} r c$ procedure available in SDTools (SD Tools, 2014). This is an iterative procedure for obtaining the poles. It starts by defining the local region where the poles might be, using a narrow band estimator. Then, the residues are estimated for the given set of poles. Finally, the poles and residues are optimized using a Non-Linear Least-Squares minimization.

The second, third and fourth columns of Table 2 show the experimental results, indicating the deformation patterns, the modal damping ratio $(\xi)$ as well as the natural frequency $\left(\omega_{n}\right)$. As can be seen in this table, values of modal damping ratio are between $1 \%$ and $1.7 \%$. 


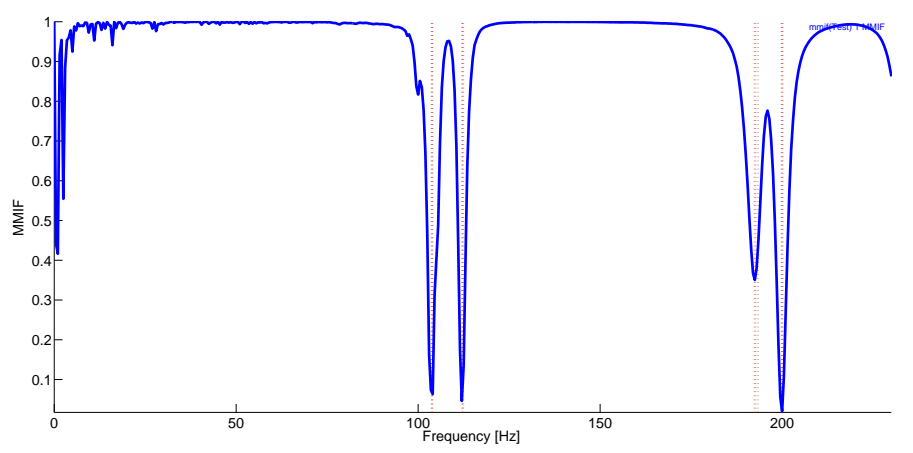

Figure 3: MMIF for pole identification

\section{Finite Element Models And Analysis}

Once the results of the experimental analysis were obtained, the numerical model was created. Initially a FE model of the sample was developed, which was then adjusted with the experimental results. Then, another model of the framework was developed with real size and boundary conditions, which was analyzed under different loading conditions. Finally, a numerical model was created using the reinforced concrete framework, which was analyzed under the same conditions as the real MCS in order to compare results. The software used for creating the models is MSC Patran and the analysis code used is MSC Nastran.

\subsection{FE model and analysis of the Sample MCS framework}

The glass fiber and resin skins surrounding the sample on its six sides were meshed with shell-type QUAD4 (MSC. Nastran, 2003) elements with four nodes each. The properties of the orthotropic material are detailed in Table 1.

The EPS nucleus was meshed with solid hexahedral elements HEX8 (MSC. Nastran, 2003). The properties of the material are also shown in Table 1. As the nucleus was composed with three glued blocks, nodes were merged to simulate perfect adhesion between the parts. The meshes of the two materials are compatible between themselves and the coincident nodes, as all the elements are regular and have an edge of 50 millimeters, having been also merged.

Figure 4 shows a section of the FE model, where red elements represent the EPS nucleus and black elements are the resin skins. Moreover, it can be 
appreciated the nucleus divisions of the three EPS glued blocks.

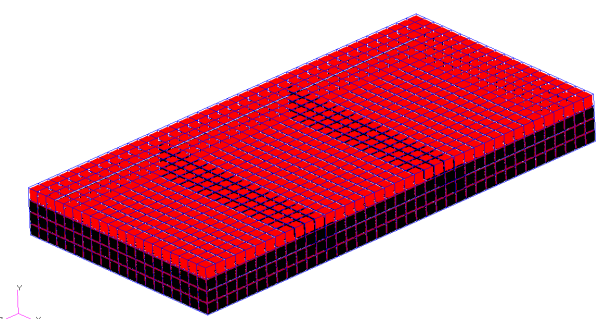

Figure 4: FE model of the sample

The simulation was performed under free body conditions. A SOL103 type process was used to calculate the modes. The natural frequencies obtained from this model are shown in the fifth column of Table 2, where they are compared with those obtained experimentally. The shapes of the modes obtained with MSC Nastran are shown in Figure 5, with a normalized scale of color.

Table 2: Deformation patterns, modal damping ratios, natural frequencies

\begin{tabular}{|c|c|c|c|c|}
\hline Mode & Def. pattern & $\xi \mathbf{( \% )}$ & $\omega_{E M A} \mathbf{( H z )}$ & $\left.\omega_{F E} \mathbf{( H z}\right)$ \\
\hline 1 & Bending & 1.42 & 103.8 & 106.2 \\
\hline 2 & Twisting & 1.14 & 112.1 & 109.9 \\
\hline 3 & Bending & 1.70 & 193.4 & 170.1 \\
\hline 4 & Twisting & 1.05 & 199.9 & 194.7 \\
\hline
\end{tabular}

Correlation between EMA and FE model of the sample framework is discussed in terms of frequency and mode shapes. Regarding the frequency, the first four natural frequencies are reasonably similar. The biggest discrepancy can be found in the third one, which presents a bending shape. This might be as a result of slight changes in the thickness of the skins, because this sample was specifically built for testing purposes. With respect to the mode shapes, the Modal Assurance Criterion (MAC) (Ewins, 2000) is applied in order to compare them. Figure 6 shows the graphical representation of the MAC. As can be seen, a good correlation between mode shapes is achieved.

\subsection{FE model and analysis of the real-dimensioned MCS framework}

The real-dimensioned MCS framework, whose size is $5 \times 3 \times 0.2$ meters, was used in the construction of a two-story prototype building, which is 


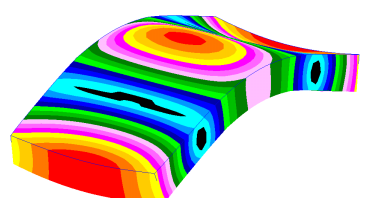

(a) Mode 1

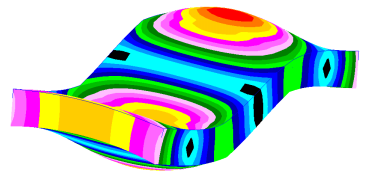

(c) Mode 3

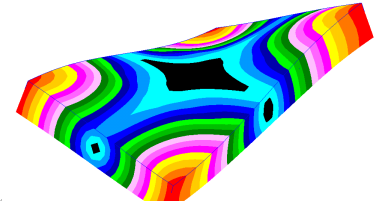

(b) Mode 2

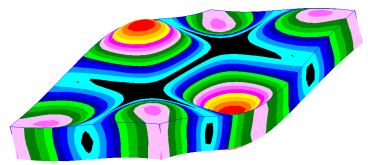

(d) Mode 4

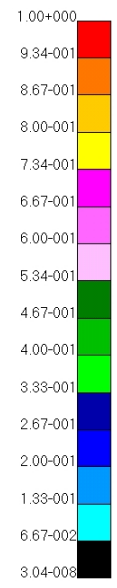

Figure 5: Four first mode shapes of the sample MCS framework obtained in the FE analysis

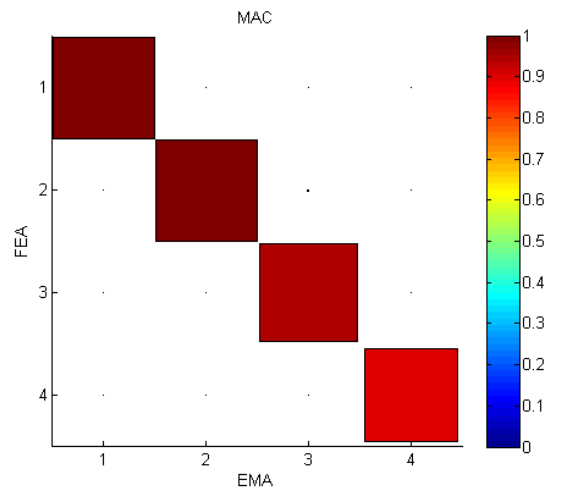

Figure 6: MAC of EMA and FE model

made of concrete columns and perimeter beams. The MCS framework lays on 150-millimeter width metallic plates that are attached to the perimeter beams. Thus, the only variation with respect to the model described in the previous subsection, apart from the scale factor, would be the following boundary conditions:(a) the framework will be supported on the metallic plates, so a perimeter of $150 \mathrm{~mm}$ on the bottom side in which the vertical displacement is constrained will be assumed; (b) the lateral faces of the framework are in contact with the perimeter beams that impede its horizontal outwards displacement. The boundary supporting elements in the prototype 
building have a high stiffness that allows for the isolated consideration of the composite framework. This approach is widely used, as for example in the work by Mello et al. (2008). Under these conditions two types of modal analyses were carried, whose results are shown in Table 3.

Table 3: Natural frequencies of the real-dimensioned MCS framework obtained from the FE analysis

\begin{tabular}{|c|c|c|}
\hline Mode & Analysis 1 $\mathbf{( H z )}$ & Analysis 2 $\mathbf{( H z )}$ \\
\hline 1 & 42.34 & 11.9 \\
\hline 2 & 50.06 & 15.72 \\
\hline 3 & 75.94 & 21.23 \\
\hline 4 & 78.89 & 22.05 \\
\hline
\end{tabular}

Analysis 1 is a modal analysis under the conditions described in the previous paragraph. Analysis 2 adds a live load of $250 \mathrm{~kg} / \mathrm{m}^{2}$. The shape of the modes can be seen in Figure 7.

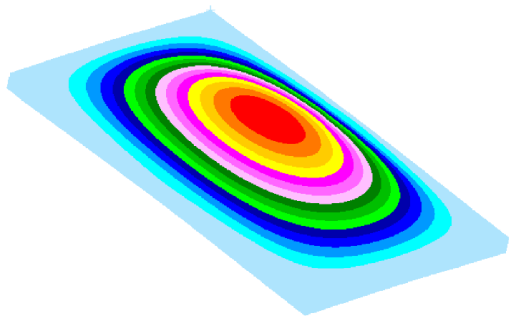

(a) Mode 1

(c) Mode 3

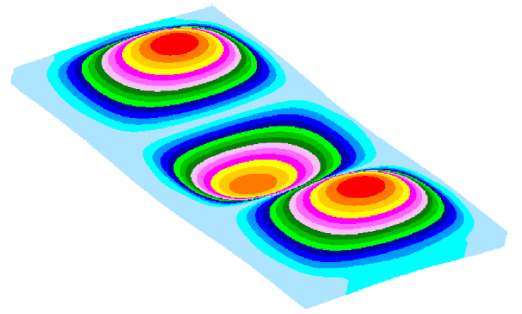

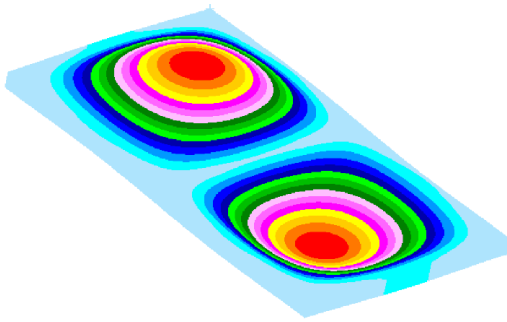

(b) Mode 2

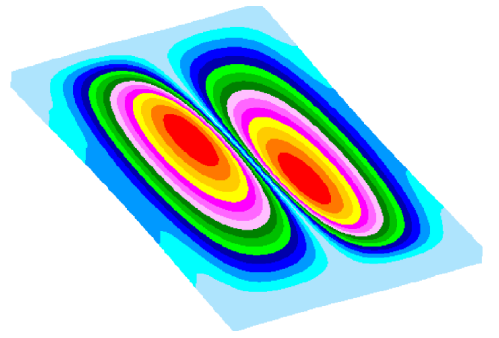

(d) Mode 4

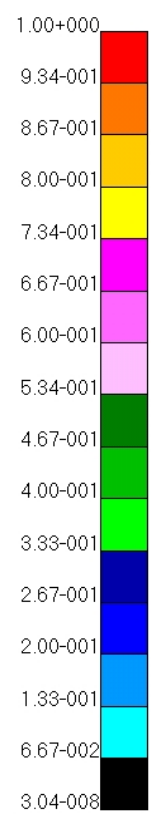

Figure 7: Four first mode deformation patterns of the real-dimensioned MCS framework obtained in the finite element analysis 
Furthermore, the response of the framework to the step of an individual in the middle was analyzed. This also includes the effect of a person stepping in the center of the framework. This test is known as the heel-drop and it consists of a person who initially is on tip toe dropping onto his/her heels (Mello et al., 2008). In the bibliography there are different models for simulating the displacement of a person over the framework. For example, a good review can be found in Mello et al. (2008), who also developed different load models which take into account the human walking dynamic effects. These vary from a sinusoidal force to a load variation model in time and position and also include the effect of the human heel impact. Živanović et al. (2007) developed a probability-based model for human-induced walking force, which takes into account variability in walking forces both induced by different pedestrians and due to imperfections in human walking.

In this case the heel-drop test was chosen as the intention was to theoretically simulate the subjective test carried out in the prototype building. The individual had a mass of $80 \mathrm{~kg}$, which was equally shared over 20 central nodes. Moreover, a force was defined over these same nodes using a ramp function with an initial value of $2760 \mathrm{~N}$ during $0.050 \mathrm{sec}$, which represents the step of the individual. As a result of this simulation under the loading conditions in Analysis 2, the graphs of displacement and acceleration were obtained for a node in the central part of the framework. They are shown in Figure 8, where the dimensions are in $\mathrm{mm}$ for the displacement and $\mathrm{mm} / \mathrm{s}^{2}$ for the acceleration.
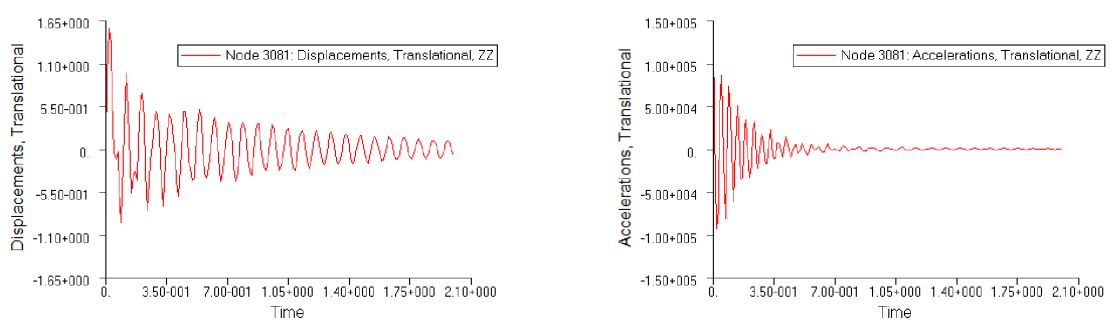

Figure 8: Heel-drop test response of the real-dimensioned MCS framework to the 'Analysis 2' conditions

\subsection{Reinforced concrete FE model and analysis}

To enable the comparison of the results of the MCS framework, another FE model was generated for Reinforced Concrete (RC), which has the same 
dimensions and conditions.

In this model, the concrete was meshed with solid hexaedrical elements HEX8 over the whole volume. The reinforcing steel had a diameter of 10 $\mathrm{mm}$ and a separation of $150 \mathrm{~mm}$, obtaining $6 \times 2$ longitudinal bars and $13 \mathrm{x}$ 2 transversal bars and were meshed with BAR2 (MSC. Nastran, 2003) bartype elements with two nodes each. A perfect adhesion was assumed between the concrete and the steel merging the common nodes. Natural frequencies under the conditions Analysis 1 and Analysis 2 are shown in Table 4.

Table 4: Natural frequencies of the reinforced concrete framework obtained from the FE analysis

\begin{tabular}{|c|c|c|}
\hline Mode & Analysis 1 $\mathbf{( H z )}$ & Analysis 2 $\mathbf{( H z )}$ \\
\hline 1 & 80.06 & 61.55 \\
\hline 2 & 113.72 & 87.34 \\
\hline 3 & 171.46 & 131.44 \\
\hline 4 & 198.79 & 152.34 \\
\hline
\end{tabular}

The response of this framework to the heel-drop test under the loading conditions in Analysis 2 are shown in Figure 9 in the form of displacement and acceleration graphs for a node in the central part of the framework. Here the dimensions are in $\mathrm{mm}$ for the displacement and $\mathrm{mm} / \mathrm{s}^{2}$ for the acceleration.
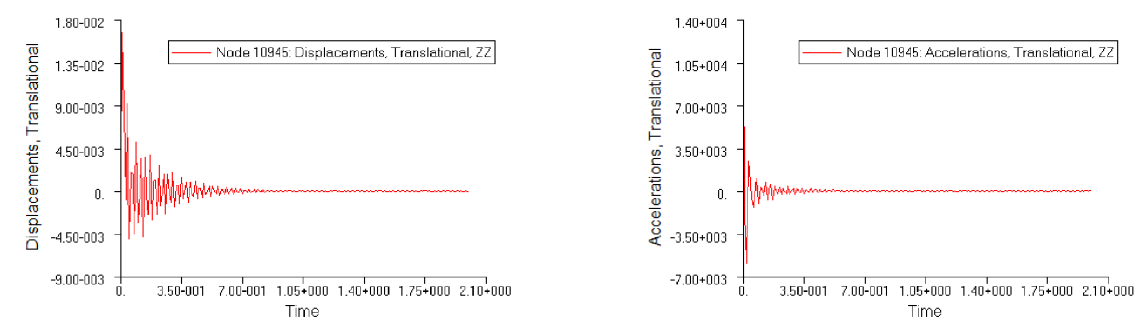

Figure 9: Heel-drop test response of the reinforced concrete framework to the 'Analysis 2' conditions

\section{Discussion}

In this section the possible causes of the lack of comfort in the composite prototype building are analyzed, which are motivated by the results obtained 
in the experimental tests and the simulation with finite elements. In this way, the problem has two variants: (a) the static aspect, in which the design criteria and resistance calculation of the frameworks are involved, and (b) the dynamic aspect, where a dynamic amplification problem is dealt with. Next, the proposed solutions for the two problems are justified.

To design the framework from the static viewpoint, the criteria of maximum stress and deformation given in the technical codes (Eurocode, 2002) have been followed. In the case of the MCS framework, the condition of maximum deformation was used, which is the most restrictive. This criterion establishes that the total deformation of the framework $\Delta_{f r m}$, which depends on the load $(P)$ and the bending stiffness $(E I)$, must be less than a particular limit value, as is demonstrated in the following expression

$$
\Delta_{f r m}=\left(P_{d l}, P_{l l}, E I\right)=\Delta_{d l}, \Delta_{l l}<\Delta_{a d m}
$$

Where $\Delta_{d l}$ and $\Delta_{l l}$ are the deformations due to the dead loads and the live loads respectively, $P_{d l}$ and $P_{l l}$ are the loads of the weight itself and the distinct live loads respectively, $E$ is Young's modulus and $I$ is the moment of bending inertia. With the criterion used in the problem analyzed in this article, it has been assumed that the total deformation must be the same for both the case of the reinforced concrete framework and the MCS, that is

$$
\Delta_{f r m}^{M C S}=\Delta_{f r m}^{R C}
$$

The dead load of the reinforced concrete itself is much greater than live load. Nevertheless, in the MCS case, these loads are of the same order of magnitude or even less than the live load. This fact has two direct consequences: (a.1) When the live loads are introduced, the deformation undergone by the concrete framework is very small with respect to that produced in the MCS, and (a.2) the total load supported by the reinforced concrete framework is of the order of twenty times greater than the total load supported by the MCS, which implies that, under the same deformation, the bending stiffness necessary in the concrete must be greater than that of MCS, as is shown in the following expression

$$
\left.\begin{array}{l}
P_{d l}^{R C}>>P_{l l} \\
P_{d l}^{M C S} \approx P_{l l}
\end{array}\right\} \Rightarrow P_{d l}^{R C}+P_{l l}>>P_{d l}^{M C S}+P_{l l} \Rightarrow E I^{R C}>>E I^{M C S}
$$

Therefore, to maintain the same deformation, if the load is increased, it is only possible to act on the product $E I$, as is indicated in expression (3). Of 
these two parameters that affect the bending stiffness, it is easier to modify the value of the moment of inertia $I$, given that Young's modulus $E$ is a material property. Thus, from the static viewpoint, it would be recommendable to increase the moment of inertia so as to reduce the static deformation. However, a simple way to modify material properties would be to use carbon stiffeners between the skins, which would constitute a design modification that would lead to further studies.

From the dynamic viewpoint, two consequences are also produced: (b.1) the fact that a person moves on such a light-weight framework means that the distribution of the mass over it is also modified appreciably, which varies the vibration modes and (b.2) observing the data in Tables 3 and 4, the influence of the mass of the live load on the first natural frequency in the case of the MCS reduces it by nearly four times, while for the concrete by only 1.3 times. As the overload increases on the MCS framework, the first natural frequency gets closer to the frequency of excitation produced by a person walking. For this reason, a problem of dynamic amplification might occur, something which does not happen in the concrete framework and thus does not involve a dynamic analysis for the RC framework. As can be observed in Figure 10, the problem of dynamic amplification depends strongly on the relative damping ratio, so that from a dynamic viewpoint, it is recommendable to increase its value in the MCS framework to improve its comfort. It should be taken into account that the value of the modal damping ratio obtained in this work is given only by the framework. This means that this value could increase when flooring, furniture, false ceiling etc. are assembled, and even it could reach the recommended value for office buildings, i. e., between $2 \%$ and $5 \%$. Hui and $\mathrm{Ng}$ (2007) propose a set of design features in order to obtain optimum vibration insulation: a small floor panel with high stiffness-to-mass ratio is recommended to increase the natural frequency; between the floor panel and the framework a honeycomb floor panel and insulators should be installed, which should be positioned at common nodal points of the two lowest symmetric bending modes of the floor panel.

These recommendations are intended to maintain the lightness of the framework as the mass is not modified, given that the principal advantage of the utilization of this type of construction material is its low fabrication and transport costs. 


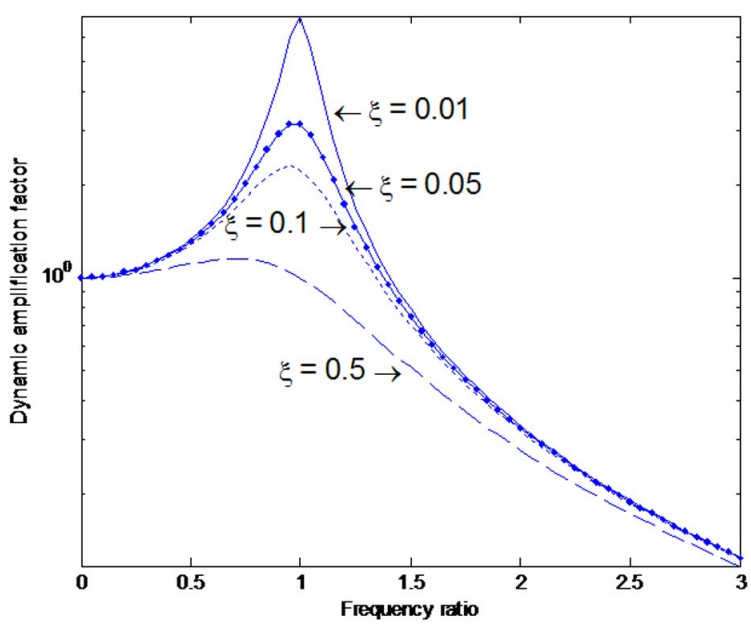

Figure 10: Dynamic amplification factor

\section{Conclusions}

The vibration response of a material-composed sandwich framework is analyzed in this paper in order to explain the lack of comfort suffered in a prototype building. The first four natural frequencies and vibration modes of the MCS framework have been determined. Firstly, an experimental modal analysis of a sample of the framework was carried out, where the damping was determined. This value of damping is within the range of a concrete framework that can be found in the literature. Then, a numerical finite element model of the sample was adjusted with the experimental results. Finally, a numerical model of the real-dimension MCS framework was built, including both live loads and boundary conditions. Another FE model of a reinforced concrete framework was built in order to compare results.

The analysis of this problem requires studying two aspects: static and dynamic behavior. From the static aspect, it was concluded that the bending stiffness of the MCS framework is too small. Thus, in order to increase this parameter, it is necessary to modify the bending stiffness product $E I$. This is because the aim is to reduce the deflection due to live loads, and so, to reduce the total deflection.

On the other hand, the analyses carried out for the MCS frameworks led to similar values of the modal damping ratios that can be found in literature for RC framework. However, the first natural frequency for the MCS frame- 
work with live loads gets closer to the excitation frequency due to pedestrians. For this reason, this could lead to a dynamic amplification problem. In the case of the reinforced concrete framework, this problem does not appear, so it is not considered. The dynamic amplification problem implies the necessity of a dynamic analysis in the case of the MCS framework. For this particular case, the solution comes from increasing the modal damping ratio although when the building is finished using flooring, ceiling and other solutions the damping might reach higher values. Furthermore, if the stiffness is increased, natural frequencies will be also increased, and the amplification dynamic problem is less important.

\section{Acknowledgement}

The authors would like to acknowledge Project DPI2013-44860 funded by the Spanish Ministry of Science and Technology and COST ACTION TU 1105 for supporting this research.

\section{References}

Altintas, G., Goktepe, A. B., 2007. The effects of orthotropic materials on the vibration characteristics of structural systems. Mechanics Based Design of Structures and Machines 35 (4), 363-380.

Augusztinovicz, F., 2012. Vibro-acoustic analysis of the stage floor of a concert hall a case study. Applied Acoustics 73 (67), 648-658.

Bluyssen, P. M., 2010. Towards new methods and ways to create healthy and comfortable buildings. Building and Environment 45 (4), 808-818.

Dooms, D., Degrande, G., Roeck, G. D., Reynders, E., 2006. Finite element modelling of a silo based on experimental modal analysis. Engineering Structures 28 (4), 532-542.

Eurocode, 2002. Basis of structural design en 1990:2002.

Ewins, D., 2000. Modal Testing: Theory Practice and Application. Research Studies Press Ltd.

Griffin, M., 1996. Handbook of Human Vibration. Elsevier. 
Hui, C. K., Ng, C. F., 2007. New floating floor design with optimum isolator location. Journal of Sound and Vibration 303 (1-2), 221-238.

ISO 2631/2, 2003. Evaluation of human exposure to whole-body vibration. continuous and shock-induced vibration inbuildings (1 to $80 \mathrm{hz}$ ).

Ljunggren, F., 2006. Floor vibration. Dynamic properties and subjective perception. PhD Tesis.

Ljunggren, F., Simmons, C., Hagberg, K., 2014. Correlation between sound insulation and occupants' perception - proposal of alternative single number rating of impact sound. Applied Acoustics 85, 57-68.

Matter, M., Gmür, T., Cugnoni, J., Schorderet, A., 2009. Numericalexperimental identification of the elastic and damping properties in composite plates. Composite Structures 90 (2), 180-187.

Mello, A. V. A., da Silva, J. G. S., da S. Vellasco, P. C. G., de Andrade, S. A. L., de Lima, L. R. O., 2008. Dynamic analysis of composite systems made of concrete slabs and steel beams. Journal of Constructional Steel Research 64 (10), 1142-1151.

Moreira, R. A. S., Rodrigues, J. D., 2010. Static and dynamic analysis of soft core sandwich panels with through-thickness deformation. Composite Structures 92 (2), 201-215.

MSC. Nastran, 2003. User's guide.

Mucchi, E., 2013. On the sweet spot estimation in beach tennis rackets. Measurement 46 (4), 1399-1410.

Pavić, A., Reynolds, P., 2003. Modal testing and dynamic fe model correlation and updating of a prototype high-strength concrete floor. Cement and Concrete Composites 25 (7), 787-799.

Petrone, G., D’Alessandro, V., Franco, F., Mace, B., Rosa, S. D., 2014. Modal characterisation of recyclable foam sandwich panels. Composite Structures 113, 362-368.

SD Tools, 2014. Structural Dynamics Toolbox \& FEMLink. User's Guide. 
Živanović, S., Pavić, A., Reynolds, P., 2007. Probability-based prediction of multi-mode vibration response to walking excitation. Engineering Structures 29 (6), 942-954.

Whittle, N., Peris, E., Condie, J., Woodcock, J., Brown, P., Moorhouse, A. T., Waddington, D. C., Steele, A., 2015. Development of a social survey for the study of vibration annoyance in residential environments: Good practice guidance. Applied Acoustics 87 (0), 83 - 93.

Williams, R., Crowley, J., Vold, H., 1985. The multivariate mode indicator function in modal analysis. International Modal Analysis Conference, 6670 .

Wu, J.-J., 2004. Finite element modelling and experimental modal testing of a three-dimensional framework. International Journal of Mechanical Sciences 46 (8), 1245-1266. 